\title{
Perspectives about Kidney Transplantation
}

\author{
Hasan MJ
}

\section{Introduction}

Kidneys are the most important and vital organs of human body. It filters wastes and excess fluids from blood, which are then excreted with urine. When kidneys lose their filtering capabilities, fluid, electrolytes and wastes can build up in patient's body. Patients with end-stage renal disease (ESRD) or chronic kidney disease (CKD) (the gradual loss of kidney function) reaches an advanced state of kidney dysfunction. Followings are some factors that may increase the risk of chronic kidney disease include:

- Diabetes

- High blood pressure

- Obesity

- Heart and blood vessel disease

- Smoking

- Family history of kidney disease

- Abnormal kidney structure

- Older age

Recently available treatments for patients with advanced CKD or ESRD are Kidney transplantation and long-term dialysis. ${ }^{1}$

Now a days Organ transplantation is one of the major developments of medical sciences. It is a life-saving process for people who are suffering from organ failures. To cure many of the diseases that growing with time, the culture of organ transplantation is not well established in the country.

It is ideal for a patient if he/she get preemptive kidney transplantation as theacute rejection is lower and increase allograft and patient survival. $^{2}$ It is not always possible for a preemptive kidney transplant for many reasons including, lack of health insurance, race/ethnicity, education level of patients, socioeconomic status, healthcare facility, diabetes etc. It is also well established that, patient survival and quality of life after kidney transplantation is far better when compared to being on dialysis. ${ }^{3}$ In Bangladesh, it is estimated that around 20 million are suffering from kidney diseases. More than 35,000 people die because of kidney failure. The yearly need for kidney transplantation is estimated at roughly 5000. However, not more than 1000 people can have kidney transplants with kidneys donated from their relatives. ${ }^{4,5}$

A kidney transplant is a surgical procedure to replace a non-functional kidney with a healthy kidney from a living or deceased donor into a person whose kidneys not functioning properly. Family members or others, who is a good match, can donate one of their kidneys. This is called a living transplant procedure and people who donate a kidney can live healthy lives with one healthy kidney. Inside the receiver, the diseased kidneys are usually left in place. The transplanted kidney is placed in the lower belly on the front side of the body.

The first successful kidney transplantation was conducted at Bangabandhu Sheikh Mujib Medical University (BSMMU) in the year 1982. From that, regular kidney transplantations have been continued from healthy donors on limited scale. ${ }^{6}$

From September 2006 to till now, around 500kidney transplantation was successfully performed with $98 \%$ success rate in Kidney Foundation Bangladesh. Here, the overall expenditure of surgery and postoperative care is around $2,60,000 \mathrm{tk}^{7}$

1. Dr. Mahmud Javed Hasan, Associate Professor and Head, Dept. of Nephrology, Community Based Medical College Bangladesh, Mymensingh.

\section{Address of correspondence:}

Email:dr.porag@gmail.com

Mobile: 01712177065 


\section{Common complications after kidney transplantation:}

The most commonly reported complications of post-kidney transplant that are usually caused by immune-mediated pathologies and nonimmunological. The most common immunological complication is allograft rejection. However, the graft rejection rate were significantly reduced due to induction of the immunosuppressive drugs but drugrelated complications have emerged as posttransplantation DM, malignancies, cardiovascular diseases, and infections. ${ }^{8}$ Many risk factors reported previously such as obesity, ethnicity, hypomagnesemia, cytomegalovirus (CMV), and hepatitis $\mathrm{C}$ viral (HCV) infections. Besides, HCV, CMV, and $B K$ virus were the most common infections after kidney transplantation. ${ }^{9}$

\section{Risk of cancer}

Previous investigations regarding kidney transplantation have demonstrated that posttransplantation infection related malignancies have been frequently noticed. The most common post-transplant carcinomas, including renal cell carcinoma, squamous cell cancer of the lip and skin, salivary gland cancer, and cholangio-carcinoma. According to previous studies, patients have a higher risk of developing colorectal and lung cancers than the general population after kidney transplantion. ${ }^{10}$

\section{Benefits of kidney transplant}

- Kidney transplant is the BEST treatment for end stage kidney disease

- Need no dialysis

- Feeling more active

- Fewer restrictions on diet

- Improves quality of life significantly for patients and their families

- Improvement in related illnesses and complications that result from end stage kidney disease such as:

$>$ High blood pressure

$>$ Anemia

$>$ Bone disease (no more phosphorus binders)

$>$ Diet restrictions

\section{Living with kidney transplant}

The following lifestyle advice is usually recommended to help one stay healthy after kidney transplantation

- Be a non-smoker and do not take alcohol

- Balanced diet (according to doctor's advice)

- Exercise and maintain body weight

$>$ Patient should try to do regular physical activity like fast walking, riding a bike on level ground, swimming etc. after six weeks of surgery and must stay at healthy weight.

$>$ Avoid lifting, pushing, or pulling heavy objects.

- Use of immunosuppressants and avoid infections

- Patient usually needs to take immunosuppressant medications for long term to prevent body's immune system from attacking the new kidney. As for this, some extra precautions needed to avoid infection like: practice good personal hygiene, avoid contact with people you know currently have infections

- Routine medical checkup.

Kidney transplantation has many advantages as it can treat the kidney failure, improve patient's health, and provide a dialysis free lifestyle. Usually, patient will have fewer fluid and some diet restrictions after surgery. But most people even feel well enough to return to work.

\section{References:}

1. Jay $C L$, Butt $Z$, Ladner DP, Skaro Al, Abecassis MM: A review of quality of life instruments used in liver transplantation. J Hepatol 2009,51(5):949-959.

2. Kasiske BL, Snyder JJ, Matas AJ, Ellison MD, Gill JS, Kausz AT. Preemptive kidney transplantation: the advantage and the advantaged. J Am SocNephrol 2002; 13: 13581364. 
3. Tonelli M, Wiebe N, Knoll G, Bello A, Browne $S$, Jadhav D, Klarenbach S, Gill J. Systematic review: kidney transplantation compared with dialysis in clinically relevant outcomes. Am J Transplant. 2011; 11: 2093-2109

4. Rahman M, Mahmood S. Status of Organ Donation and Transplantation in Bangladesh. Transplantation. 2017;101:8S-2.

5. Billah MM, Farzana $H$, Latif $A$, Mitra $P$, Chowdhury TA, Rahim MA, et al. Knowledge and attitude of Bangladeshi physicians towards organ donation and transplantation. Bangladesh Critical Care Journal. 2016;4(1):23-7.

6. Ali M. Organ Transplantation in Bangladesh Challenges and Opportunities. Ibrahim Medical College Journal. 2012;6(1):i-ii.

7. https://www.kidneyfoundationbd.com/facilities /transplantation-facility

8. Al-Amri TM, Alharthi YH, Alkhamis AS, Muhaif AlA, Almutairi MA, Mousa NMA, et al. Common complications post-kidney transplantation: a literature review. Int $\mathrm{J}$ Community Med Public Health 2021;8

9. Baid-Agrawal $S$, Pascual $M$, Moradpour D, Somasundaram R, Muche $M$. Hepatitis $C$ virus infection and kidney transplantation in 2014: what's new? Am J Transplant. 2014;14(10):2206-20.

10. Engels EA, Pfeiffer RM, Fraumeni JF. Spectrum of cancer risk among US solid organ transplant recipients. JAMA. 2011;306(17):1891-901. 